\title{
A Pilot Study of Ponatinib in Patients With FGFR- Altered Advanced Cholangiocarcinoma
}

\section{Daniel H. Ahn}

Mayo Clinic Scottsdale: Mayo Clinic Arizona

\section{Pedro Luiz Serrano Uson Junior}

Mayo Clinic Scottsdale: Mayo Clinic Arizona

\section{Peter Masci}

Mayo Clinic Scottsdale: Mayo Clinic Arizona

Heidi Kosiorek

Mayo Clinic Scottsdale: Mayo Clinic Arizona

Thorvardur R. Halfdanarson

Mayo Clinic Rochester

Kabir Mody

Mayo Clinic's Campus in Florida

Hani Babiker

Mayo Clinic's Campus in Florida

Thomas DeLeon

Mayo Clinic Arizona

\section{Mohamad Bassam Sonbol}

Mayo Clinic Arizona

\section{Gregory Gores}

Mayo Clinic Rochester

\section{Rory Smoot}

Mayo Clinic Rochester: Mayo Clinic Minnesota

\section{Tanios Bekaii-Saab}

Mayo Clinic Arizona

\section{Amit Mahipal}

Mayo Clinic Rochester: Mayo Clinic Minnesota

\section{Aaron Mansfield}

Mayo Clinic Rochester

Nguyen H Tran

Mayo Clinic Rochester

Joleen M Hubbard

Mayo Clinic Rochester

Mitesh J. Borad ( $\square$ borad.mitesh@mayo.edu ) 


\section{Research Article}

Keywords: next generation sequencing, targetable mutations, bile duct cancers, cholangiocarcinoma, FGFR, ponatinib

Posted Date: July 29th, 2021

DOI: https://doi.org/10.21203/rs.3.rs-741191/v1

License: (1) This work is licensed under a Creative Commons Attribution 4.0 International License. Read Full License 


\section{Abstract}

\section{Background:}

Biliary tract cancers (BTC) are rare, chemo resistant and are associated with a poor prognosis. Preclinical and early clinical work had demonstrated interesting anti-tumor activity from targeting fibroblast growth factor receptor (FGFR) pathway. We hypothesized that ponatinib, a multi-targeted tyrosine kinase inhibitor with activity against FGFR, would be active in BTC patients with FGFR alterations.

\section{Methods:}

This was a multi-center, single institution pilot study of ponatinib in patients with advanced, refractory BTC with FGFR alterations. The primary end point was overall response rate, with secondary points of overall survival (OS), progression-free survival (PFS) and Health Related Quality of Life (HRQoL) assessment.

\section{Results:}

Twelve patients were enrolled prior to early termination of the trial. Clinical benefit (defined as complete/partial response + stable disease) of $45.5 \%$ was observed. All observed toxicities were manageable and reversible. Toxicities were mild, with lymphopenia (75\%), rash (63\%) and fatigue (50\%) being the most frequent. No significant detriment in global QoL was observed.

\section{Conclusions:}

Ponatinib as a single agent in FGFR altered BTC is tolerable with very modest clinical activity. This is the first report of prospective assessment of FGFR inhibition in BTC using ponatinib, and the first study to report its effect on HRQoL. Further development of ponatinib will involve correlative studies to better refine patient selection, focus on combinations with other molecular targeted agents, conventional cytotoxic chemotherapy, and studies to better understand mechanisms of treatment resistance.

\section{Introduction}

Biliary tract cancers (BTC) comprise malignant tumors of the intrahepatic and extrahepatic ducts, and gallbladder [1,2]. The majority of patients present with advanced disease, where the standard treatment entails the combination of gemcitabine and cisplatin cytotoxic chemotherapy [3]. While the combination resulted in a median 3.6-month survival benefit and $80 \%$ disease control rate compared to gemcitabine monotherapy, all patients eventually succumb to treatment resistance, where the overall survival (OS) remains less than 1 year [3]. Patient outcomes in second-line therapies in clinical trials after gemcitabine platinum-based chemotherapy failure resulted in limited outcomes with an incremental improvement in progression free survival (PFS) and OS, emphasizing the urgent need to develop novel effective treatments in BTC [4-10]. 
The availability and implementation of next-generation sequencing has allowed us to identify the presence of tumor somatic variants and its functional consequence on tumor growth, treatment resistance and prognosis. Importantly, this development has provided the rationale to develop targeted therapies against these genetic alterations. The fibroblast growth factor receptors (FGFR) are a family of highly conserved tyrosine kinase receptors consisting of four members (FGFR1-4). These receptors bind to secreted glycoprotein ligands (fibroblast growth factor) to their extracellular domain [11]. FGFR alterations occur in $~ 15 \%$ of intrahepatic cholangiocarcinoma (iHCC) with the vast majority being translocations [12, 13]. A broad array of FGFR2 chromosomal fusions with various genomic partners have been identified in iHCC [14-19]. Preclinical studies have identified that the fusion with the tyrosine kinase protein results in the activation of the FGFR2 receptor with autophosphorylation and activation of downstream signaling pathways ( $R A S / M A P K, P I 3 K / A K t$ and STAT pathway), which regulate cellular growth, differentiation, and apoptosis [20]. FGFR inhibition represents a promising therapeutic strategy in patients with FGFR mutant BTC. FGFR specific small molecule inhibitors have now undergone evaluation in several pivotal single arm studies and exhibited response rates ranging from $23 \%$ to $37 \%$ [21-23].

In this study, to evaluates the potential anti-tumor activity from FGFR inhibition, BTC patients with FGFR alterations that progressed on conventional chemotherapy were given ponatinib ${ }^{24}$. Ponatinib is an orally bioavailable multi-target tyrosine kinase inhibitor that inhibits all members of the FGFR family, along with several other kinases including KIT, RET, SRC, VEGFR and PDGFR [24]. Based on the preliminary observed anti-tumor activity [24], we conducted a pilot trial to investigate the anti-tumor activity from ponatinib in patients with advanced BTC with FGFR2 alterations. Here in this report, we outline the findings from this study.

\section{Patients And Methods}

The protocol for the study was reviewed and approved by the Mayo Clinic Institutional Review Board. The study procedures were carried out in accordance with the approved guidelines and regulations. Eligible patients were required to have histologically or cytologically confirmed advanced biliary tract cancer refractory or intolerant to gemcitabine or fluoropyrimidine based chemotherapy with FGFR2 fusion/rearrangement (determined utilizing either a next generation sequencing assay or fluorescent in situ hybridization (FISH) or FGFR mutation/amplification. Assays were required to be performed in a CLIA certified laboratory and done as a CLIA validated test or RUO in a CLIA laboratory. All patients provided written informed consent before the initiation of the study.

Patients were also required to have evidence of measurable or evaluable disease according to Response Evaluation Criteria in Solid Tumors (RECIST) version 1.1 [25], an Eastern Cooperative Oncology Group performance status of 0-2 [26]. Patients were required to meet pre-specified parameters for acceptable organ function, including total bilirubin $\leq 1.5$ times the upper limit of normal and AST/ALT $\leq 2.5$ times the upper limit of normal. 
Selected exclusion criteria included cancers of Ampulla of Vater, prior or current treatment with a selective FGFR inhibitor, current significant corneal or retinal disorder/keratopathy, history and/or current extensive tissue calcification or alterations in calcium or phosphate homeostasis, gastrointestinal impairment or disease that could alter ponatinib absorption, concurrent treatment with strong cytochrome P450 3A4 inducers/inhibitors, intake of foods with potential CYP3A4 interaction, or drugs or supplements that increase phosphorous or calcium levels, and participation in concurrent investigational drug or device studies.

\section{Study Design}

This was a multi-center, single institution, open label, single arm pilot study that was conducted at the Mayo Clinic (Rochester, Arizona, and Florida). Tumor response to ponatinib was assessed per RECIST version 1.1 using computed tomography or magnetic resonance imaging. Adverse events were assessed according to the Common Terminology Criteria for Adverse Events (CTCAE), version 4.0 [27], during treatment and until 30 days after the last dose was administered. Health-related quality of life (HRQoL) was evaluated using European Organization for Research and Treatment of Cancer (EORTC) Quality of Life Questionnaire-C30 version 3.0 and the biliary tract cancer specific EORTC Quality of Life Questionnaire (QLQ) BIL-21 [28, 29].

\section{Statistical Analysis}

This study was designed using Simon's two-stage approach. The true overall response rate was set at $5 \%$ and $20 \%$ under null and alternative hypotheses, respectively. The primary endpoint of the study was clinical benefit rate, which was defined as confirmed tumor response or stable disease for 4 or more cycles. Each cycle consisted of 28 days in length. A confirmed tumor response was defined to be either a complete response (CR) or partial response (PR) noted as the objective status on 2 consecutive evaluations at least 8 weeks apart. If 1 or more clinical responses were seen from the first 10 patients, the study would proceed to the second stage. If three or more responses were observed in the first 27 evaluable patients, the agent would be considered promising with consideration for further evaluation in subsequent studies.

Secondary endpoints included progression free survival (PFS), overall survival (OS), and time to definitive deterioration (TDD) of HRQoL and adverse event profile of ponatinib. Safety assessments included physical examination, performance status assessment (according to ECOG), clinical laboratory values and concomitant medication use. All observed toxicities and side effects were graded according to $\mathrm{NCl}$ CTCAE 4.0. Skin and bowel symptoms were assessed using the Skindex-15 and Bowel Function Questionnaire (BFQ). The Skindex-16 contains 16 questions each on a 0-10 scale ( $0=$ never bothered, $10=$ always bothered) assessing skins problems and has previously been validated [30] . The BFQ, developed and created by Mayo Clinic physicians, identifies patient-reported problems with various aspects of bowel function (yes or no response) to help evaluate how bowel dysfunction affects normal activities and quality of life (QOL). Content validity of this instrument has been previously reported [31]. 
QoL assessments were conducted at baseline and every cycle during follow up and scored according to the published scoring algorithm. Scale score trajectories over time were examined using stream plots and mean plots with standard deviation error bars overall. TDD of HRQoL was defined as the time between randomization and worsening of global, physical functioning, or fatigue, based on changes from baseline, which were statistically tested using paired t-tests, and standardized response means (mean of the change from baseline scores at a given cycle, divided by the standard deviation of the change scores) were interpreted [after applying Middel's (2002) adjustment] using Cohen's (1988) cut-offs: $<0.20=$ trivial; $0.20<0.50=$ small; $0.50-<0.80=$ moderate and $>=/ 0.80=$ large. Correlation between outcomes employed Pearson and/or Spearman correlations at individual time points.

\section{Results}

Twelve patients were enrolled from December 1, 2014, through July 31, 2017. The trial completed the interim analysis but was terminated prior to the completion of second stage, given the advent of more FGFR specific inhibitors and modest anti-tumor efficacy observed in this initial set of patients. Patient characteristics are listed in Table 1. Of the 12 patients enrolled, all 12 were evaluable for toxicity and 11 were evaluable for response (one patient did not desire to pursue further treatment and was not evaluable for response at the first restaging scan at 8 weeks) (Table 2). Of the 12 patients that were enrolled into the study, most patients were female (75\%), white (83.3\%) and with intrahepatic cholangiocarcinoma (83.3\%). All patients had measurable disease, and most patients' tumors harbored FGFR2 translocations (83.3\%).

\section{Treatment Toxicity}

Most patients (58.3\%) were able to tolerate full dose without requiring any dose modifications. Four dose modifications were due to cutaneous toxicities, while an additional 3 were due to non-hematologic toxicities. The most observed toxicities (Table 3 ) were fatigue, abdominal pain, and constipation. Toxicities were primarily grade 1 or 2 and were reversible. Only 1 patient experienced a grade 4 toxicity (sepsis), which was deemed unrelated to ponatinib. All observed toxicities were manageable and reversible.

\section{Treatment Efficacy}

A median number of 4 cycles was administered per patient (range, 1-10). One patient (9.1\%) experienced a partial response with 4 (36.4\%) patients that experienced stable disease (Table 2). Most patients discontinued therapy due to disease progression (83.3\%), Discontinuation in one patient was due to a severe adverse event (8.3\%) and in another was on account of desire to not pursue further treatment. Median PFS was 2.4 months (Figure 1A) with a median OS of 15.7 months (Figure 1B). A Spider plot presenting duration and tumor changes from baseline for each subject can be seen on Figure 2 .

\section{HRQoL Assessment}


All twelve patients completed the HRQoL questionnaires at baseline, 9 at cycle 2, 5 at cycles 3 and 4, and 4 at cycle 5 (Supplementary table 1). For the EORTC QLQ C30, no difference in time to deterioration of global HRQoL was observed. There was no significant difference in physical functioning, role functioning, emotional functioning, social functioning, and cognitive functioning (Supplementary figures 1-6). For the EORTC BIL-21, worsening in tiredness (fatigue) was observed and coincided with treatment duration (Supplementary figure 7). No differences in skin from Skindex-16 or bowel function were observed (Supplementary figures 8-11).

\section{Discussion}

Advanced BTC remains a challenging cancer with a universally poor prognosis with limited treatment options $[32,33]$. The rationale for FGFR inhibition was based on the integral role of FGFR signaling pathways with tumorigenesis, treatment resistance and disease progression in BTC. Findings from our previous pre-clinical and early studies demonstrated anti-tumor activity from FGFR inhibition in FGFR mutated BTC cell lines and patients, suggesting the potential for targeting FGFR as a potential therapy for patients whose tumors exhibit FGFR alterations [24].

This pilot study evaluated the efficacy of ponatinib, a multi-targeted tyrosine kinase inhibitor with activity inhibiting multiple targets including all FGFR. Our findings demonstrate the potential anti-tumor activity of ponatinib in patients with FGFR2 altered biliary tract cancer. In the limited evaluation, $45.5 \%$ of patients experienced a clinical benefit response as was pre-defined (partial response + complete response + stable disease), including one patient who experienced a partial response and two patients that experienced prolonged SD (> 12 weeks). The modest observed clinical activity from ponatinib may be in part related to its broad multi-targeted profile in comparison to more selective pan-FGFR inhibitors. Furthermore, given its multi-targeted activity and the resultant associated side effects such as thrombosis and hypertension, the more modest anti-tumor activity from FGFR inhibition may be a result from the potential inability to administer higher doses.

An improvement in patient selection, identifying those likely to derive the most benefit from FGFR inhibitors, could have resulted in a greater proportion of patients deriving anti-tumor activity. Patients that experienced a clinical response from ponatinib were in those whose tumors expressed FGFR2 translocations, whereas no clinical benefit was seen in those with FGFR mutations. However, the premature termination of the trial did not allow us to fully assess the true efficacy and potential benefit of ponatinib in biliary tract cancer. While our findings have demonstrated the potential of FGFR inhibition using ponatinib in BTC, findings from other clinical trials that have investigated various selective panFGFR inhibitors in FGFR mutant BTC have demonstrated consistent clinical results. In patients with BTC whose tumors expressed FGFR2 translocations, objective response rates of up to $40 \%$ were observed [21$23,34]$, reaffirming the potential therapeutic benefit from FGFR2 inhibition in this disease.

The adverse effects observed from ponatinib were limited and reversible, with no significant detrimental effect on global HRQoL. While QLQ-BIL-21 has demonstrated its potential as a QoL assessment tool, its 
utility has not been validated for clinical use. Efforts to incorporate the QLQ-BIL-21 scale are ongoing in other FGFR inhibitor studies as well [35-37]. PROOF, a randomized phase III clinical trial comparing infigratinib, a FGFR inhibitor, to gemcitabine and cisplatin, in patients with untreated advanced BTC whose tumors harbor FGFR2 translocations, has incorporated QLQ-BIL-21 and its results will help confirm its ability as QoL assessment tool [37].

This is the first report of a prospective trial of ponatinib in FGFR-altered biliary tract cancers. Correlative investigation, including work into elucidating mechanisms of de novo and treatment-acquired resistance is needed to better develop alternative treatment strategies. In patients that were previously treated with infigratinib and futibatinib, a pan FGFR inhibitor, post progression tumor biopsies paired with circulating tumor DNA identified new point mutations in FGFR kinase domains $[38,39]$. These acquired alterations resulted in conformational changes that prevented adequate drug binding and insufficient tumor growth inhibition [38]. Additional proposed mechanisms of resistance include the secondary activation of other receptor tyrosine kinases as an escape mechanism to overcome FGFR inhibition. Interestingly, in preclinical studies ponatinib retained potency against p.E565A and p.L617M resistance mutations in FGFR2. Given that infigratinib and pemigatinib are resistant to these mutations, ponatinib could be considered for use in instances where therapeutic resistance is driven by FGFR2 p.E565A and/or p.L617M.

In conclusion, ponatinib as a single agent in biliary cancer was generally tolerable with no significant detriment on QoL, but with only modest clinical activity compared to FGFR specific small molecule inhibitors, in this pilot study. The limited anti-tumor activity from ponatinib may in part be from its multitargeted activity compared to other agents with more FGFR selectivity. If further studies for ponatinib are undertaken, they should focus on patients with resistance mutations such as the ones described earlier.

\section{Declarations}

Funding: This work was supported by the National Institute of Health $(\mathrm{NIH})$ through a DP2 Award CA195764 (to MJB); National Cancer Institute (NCI) K12 award CA090628 (to MJB), SPORE Project Award 5P50CA210964-03 (MJB), Mayo Clinic Center for Individualized Medicine (CIM) Precision Cancer Therapeutics Program; and Mayo Clinic Cancer Center. The funders had no role in study design, data collection and analysis, decision to publish or preparation of the manuscript. Its contents are solely the responsibility of the authors and do not necessarily represent the official views of the $\mathrm{NIH}$.

Conflicts of interest/Competing interests: MJB has received grant to institution from Senhwa Pharmaceuticals, Adaptimmune, Agios Pharmaceuticals, Halozyme Pharmaceuticals, Five Prime Pharmaceuticals, Celgene Pharmaceuticals, EMD Merck Serono, Toray, Dicerna, Taiho Pharmaceuticals, Sun Biopharma, Isis Pharmaceuticals, Redhill Pharmaceuticals, Boston Biomed, Basilea, Incyte Pharmaceuticals, Mirna Pharmaceuticals, Medimmune, Bioline, Sillajen, ARIAD Pharmaceuticals, PUMA Pharmaceuticals, Novartis Pharmaceuticals, QED Pharmaceuticals, Pieris Pharmaceuticals, consultancy from ADC Therapeutics, Exelixis Pharmaceuticals, Inspyr Therapeutics, G1 Therapeutics, Immunovative 
Therapies, OncBioMune Pharmaceuticals, Western Oncolytics, Lynx Group, and travel support from Astra Zeneca.

Availability of data and material: All data and materials are available for verification as needed Code availability: Not applicable

Authors' contributions: Daniel Ahn has written the manuscript and created the tables, assisted in the design, and final approval of the manuscript. Pedro Uson Junior assisted in writing the manuscript, review, and final approval of the manuscript. Mitesh Borad has assisted in the design, writing, review, and final approval of the manuscript. Peter Masci, Heidi Kosiorek, Thorvardur R. Halfdanarson, Kabir Mody, Hani Babiker, Thomas DeLeon, Mohamad Bassam Sonbol, Gregory Gores, Rory Smoot, Tanios BekaiiSaab, Amit Mahipal, Aaron Mansfield, Nguyen H Tran, Joleen M Hubbard assisted in the design, review, and final approval of the manuscript.

Ethics approval: The study was approved by the Mayo Clinic Institutional Review Board (IRB).

Consent to participate: All participants provided informed consent as outlined in the manuscript

Consent for publication: all authors approved the final version of this manuscript.

\section{References}

1. Torre LA, Siegel RL, Islami F, Bray F, Jemal A (2018). Worldwide burden of and trends in mortality from gallbladder and other biliary tract cancers. Clin Gastroenterol Hepatol 16.3: 427-437.

2. Jarnagin WR, Ruo L, Little SA, et al (2003) Patterns of initial disease recurrence after resection of gallbladder carcinoma and hilar cholangiocarcinoma: implications for adjuvant therapeutic strategies. Cancer 98:1689-1700.

3. Valle J, Wasan H, Palmer DH, et al (2010) Cisplatin plus gemcitabine versus gemcitabine for biliary tract cancer. N Engl J Med 362:1273-1281

4. Lamarca A, Palmer DH, Wasan HS et al (2021). Second-line FOLFOX chemotherapy versus active symptom control for advanced biliary tract cancer (ABC-06): a phase 3, open-label, randomised, controlled trial. Lancet Oncol 22.5: 690-701.

5. Paule B, Herelle MO, Rage E et al (2007) Cetuximab plus gemcitabine-oxaliplatin (GEMOX) in patients with refractory advanced intrahepatic cholangiocarcinomas. Oncology 72:105-10

6. Suzuki E, Ikeda M, Okusaka T, et al (2013) A multicenter phase II study of S-1 for gemcitabine-refractory biliary tract cancer. Cancer Chemother Pharmacol 71:1141-6 
7. Bridgewater J, Palmer D, Cunningham D, et al (2013) Outcome of second-line chemotherapy for biliary tract cancer. Eur J Cancer 49:1511

8. Croitoru A, Gramaticu I, Dinu I, et al (2012) Fluoropyrimidines plus cisplatin versus gemcitabine/gemcitabine plus cisplatin in locally advanced and metastatic biliary tract carcinoma - a retrospective study. J Gastrointestin Liver Dis 21:277-84

9. Fornaro L, Vivaldi C, Cereda S, et al (2015) Second-line chemotherapy in advanced biliary cancer progressed to first-line platinum-gemcitabine combination: a multicenter survey and pooled analysis with published data. J Exp Clin Cancer Res 34:156

10. Sasaki T, Isayama H, Nakai Y, et al (2013) A pilot study of salvage irinotecan monotherapy for advanced biliary tract cancer. Anticancer Res 33:2619-22

11. Belov AA, Mohammadi M (2013) Molecular mechanisms of fibroblast growth factor signaling in physiology and pathology. Cold Spring Harb Perspect Biol 5

12. Churi CR, Shroff R, Wang Y, et al (2014) Mutation profiling in cholangiocarcinoma: prognostic and therapeutic implications. PLoS One 9:e115383

13. Javle M, Bekaii-Saab T, Jain A, et al (2016) Biliary cancer: Utility of next-generation sequencing for clinical management. Cancer 122:3838-3847

14. Borad MJ, Champion MD, Egan JB, et al (2014) Integrated genomic characterization reveals novel, therapeutically relevant drug targets in FGFR and EGFR pathways in sporadic intrahepatic cholangiocarcinoma. PLoS Genet 10:e1004135

15. Ross JS, Wang K, Gay L, et al (2014) New routes to targeted therapy of intrahepatic cholangiocarcinomas revealed by next-generation sequencing. Oncologist 19:235-42

16. Sia D, Losic B, Moeini A, et al (2015) Massive parallel sequencing uncovers actionable FGFR2PPHLN1 fusion and ARAF mutations in intrahepatic cholangiocarcinoma. Nat Commun 6:6087

17. Arai Y, Totoki Y, Hosoda F, et al (2014) Fibroblast growth factor receptor 2 tyrosine kinase fusions define a unique molecular subtype of cholangiocarcinoma. Hepatology 59:1427-34

18. Wu YM, Su F, Kalyana-Sundaram S, et al (2013) Identification of targetable FGFR gene fusions in diverse cancers. Cancer Discov 3:636-47

19. Graham RP, Barr Fritcher EG, Pestova E, et al (2014) Fibroblast growth factor receptor 2 translocations in intrahepatic cholangiocarcinoma. Hum Pathol 45:1630-8

20. Kouhara H, Hadari YR, Spivak-Kroizman T, et al (1997) A lipid-anchored Grb2-binding protein that links FGF-receptor activation to the Ras/MAPK signaling pathway. Cell 89:693-702 
21. Abou-Alfa GK, Sahai V, Hollebecque A et al (2020) Pemigatinib for previously treated, locally advanced or metastatic cholangiocarcinoma: a multicentre, open-label, phase 2 study. Lancet Oncol, 21(5):671-684

22. Javle M, Lowery M, Shroff RT et al (2018) Phase II study of BGJ398 in patients with FGFR-altered advanced cholangiocarcinoma. J Clin Oncol 36.3: 276

23. Bridgewater J, Meric-Bernstam F, Hollebecque A et al (2020) Efficacy and safety of futibatinib in intrahepatic cholangiocarcinoma (iCCA) harboring FGFR2 fusions/other rearrangements: Subgroup analyses of a phase II study (FOENIX-CCA2). Ann Oncol 31: S261-S262.

24. Gozgit JM, Wong MJ, Moran L, et al (2012) Ponatinib (AP24534), a multitargeted pan-FGFR inhibitor with activity in multiple FGFR-amplified or mutated cancer models. Mol Cancer Ther 11:690-9

25. Eisenhauer EA, Therasse P, Bogaerts J, et al (2009) New response evaluation criteria in solid tumours: revised RECIST guideline (version 1.1). Eur J Cancer 45:228-47

26. Oken MM, Creech RH, Tormey DC, et al (1982) Toxicity and response criteria of the Eastern Cooperative Oncology Group. Am J Clin Oncol 5:649-55

27. Common Terminology Criteria for Adverse Events (CTCAE) (2021). In:

https://ctep.cancer.gov/protocolDevelopment/electronic_applications/ctc.htm. Accessed on July 2021

28. Kaupp-Roberts SD, Yadegarfar G, Friend E, et al (2016) Validation of the EORTC QLQ-BIL21 questionnaire for measuring quality of life in patients with cholangiocarcinoma and cancer of the gallbladder. Br J Cancer 115:1032-1038

29. Friend E, Yadegarfar G, Byrne C, et al (2011) Development of a questionnaire (EORTC module) to measure quality of life in patients with cholangiocarcinoma and gallbladder cancer, the EORTC QLQBIL21. Br J Cancer 104:587-92

30. Chren MM, Lasek RJ, Sahay AP, Sands LP (2001) Measurement properties of Skindex-16: a brief quality-of-life measure for patients with skin diseases. J Cut Med Surg 5.2: 105-110.

31. Atherton, PJ, Halyard MY, Sloan MJ et al (2013) Assessment of patient-reported measures of bowel function before and after pelvic radiotherapy: an ancillary study of the North Central Cancer Treatment Group study NO0CA." Supp Care Cancer 21.4: 1193-1199.

32. Arora M, Bogenberger JM, Abdelrahman A et al (2020) Evaluation of NUC-1031: A first-in-class ProTide in biliary tract cancer." Cancer chemother pharmacol 85: 1063-1078.

33. Uson Junior PLS, Bogenberger J, Borad MJ (2020). "Advances in the treatment of biliary tract cancers." Curr opinion gastroentero/ 36.2: 85-89. 
34. Cleary JM, lyer G, Oh DH et al (2020) Final results from the phase I study expansion cohort of the selective FGFR inhibitor Debio 1,347 in patients with solid tumors harboring an FGFR gene fusion. J Clin Oncol : 3603-3603

35. Valle JW, Bibeau K, Cho Y, et al (2021) Longitudinal evaluation of quality of life (QoL) in patients (Pts) with FGFR2-driven cholangiocarcinoma (CCA) treated with pemigatinib. J Clin Oncol 39:276-276

36. Bekaii-Saab TS, Valle JW, Van Cutsem E et al (2020) FIGHT-302: Phase III study of first-line (1L) pemigatinib (PEM) versus gemcitabine (GEM) plus cisplatin (CIS) for cholangiocarcinoma (CCA) with FGFR2 fusions or rearrangements. J Clin Oncol, 38: abstr TPS592.

37. Javle MM, Borbath I, Clarke SJ et al (2019) Infigratinib versus gemcitabine plus cisplatin multicenter, open-label, randomized, phase 3 study in patients with advanced cholangiocarcinoma with FGFR2 gene fusions/translocations: the PROOF trial. J Clin Oncol 37.15 suppl.

38. Goyal L, Saha SK, Liu LY, et al (2017) Polyclonal Secondary FGFR2 Mutations Drive Acquired Resistance to FGFR Inhibition in Patients with FGFR2 Fusion-Positive Cholangiocarcinoma. Cancer Discov 7:252-263

39. Goyal L, Shi L, Liu LY et al (2019) TAS-120 overcomes resistance to ATP-competitive FGFR inhibitors in patients with FGFR2 Fusion-Positive intrahepatic cholangiocarcinoma. Cancer Disc; 9(8): 1064-1079.

\section{Tables}

Table 1. Patient Demographics and Characteristics 


\begin{tabular}{|c|c|c|}
\hline & Total & $(\mathrm{N}=12)$ \\
\hline \multicolumn{3}{|l|}{ Age (years) } \\
\hline Median & 48.5 & \\
\hline Range & $40.0-66.0$ & \\
\hline \multicolumn{3}{|l|}{ Gender } \\
\hline Female & $9(75 \%)$ & \\
\hline Male & $3(25 \%)$ & \\
\hline \multicolumn{3}{|l|}{ Race } \\
\hline American Indian or Alaska Native & $1(8.3 \%)$ & \\
\hline Black or African American & $1(8.3 \%)$ & \\
\hline White & $10(83.3 \%)$ & \\
\hline \multicolumn{3}{|l|}{ ECOG Performance Score } \\
\hline 0 & $3(25 \%)$ & \\
\hline 1 & $7(58.3 \%)$ & \\
\hline 2 & $2(16.7 \%)$ & \\
\hline \multicolumn{3}{|l|}{ Histologic Type } \\
\hline Adenocarcinoma & $12(100 \%)$ & \\
\hline \multicolumn{3}{|l|}{ Primary tumor site } \\
\hline Gallbladder & $2(16.7 \%)$ & \\
\hline Intrahepatic cholangiocarcinoma & $10(83.3 \%)$ & \\
\hline \multicolumn{3}{|l|}{ Prior surgery } \\
\hline Yes & $7(58.3 \%)$ & \\
\hline No & $5(41.7 \%)$ & \\
\hline \multicolumn{3}{|l|}{ Prior radiation therapy } \\
\hline Yes & $7(58.3 \%)$ & \\
\hline No & $5(41.7 \%)$ & \\
\hline \multicolumn{3}{|l|}{ Prior systemic therapy } \\
\hline Yes & $12(100 \%)$ & \\
\hline
\end{tabular}


Table 2. Response assessment (Evaluable patients, $n=11$ )

\begin{tabular}{|lll|}
\hline \multicolumn{1}{|l}{ Total } & 95\% Confidence interval \\
\hline Best Response & & \\
\hline CR & 0 & - \\
PR & 1 & $9.1 \%(0.2-41.3 \%)$ \\
\hline SD* & 4 & $36.4 \%(10.9-69.2 \%)$ \\
Progression & 6 & $54.5 \%(23.4-83.3 \%)$ \\
\hline & & \\
Clinical Benefit (CR+PR+SD) * * & 5 & $45.5 \%(16.8-76.6 \%)$ \\
\hline
\end{tabular}

One patient declined further treatment and was not evaluable for response at 8 weeks.

*Two patients had a best response of SD maintained for only 2 cycles.

${ }^{\star *} \mathrm{CR}$ or PR as best response OR SD for 4 or more cycles

Table 3. Common Toxicities $(n=12)$ 


\begin{tabular}{|c|c|c|c|c|c|c|c|c|}
\hline \multirow[t]{3}{*}{ Adverse Event (Type) } & \multicolumn{8}{|c|}{ Grade } \\
\hline & \multicolumn{2}{|l|}{1} & \multicolumn{2}{|l|}{2} & \multicolumn{2}{|l|}{3} & \multicolumn{2}{|l|}{4} \\
\hline & $\mathbf{N}$ & $\%$ & $\mathbf{N}$ & $\%$ & $\mathbf{N}$ & $\%$ & $\mathbf{N}$ & $\%$ \\
\hline Rash maculo-papular & 3 & 25.0 & 2 & 16.7 & & & & \\
\hline Nausea & 4 & 33.3 & & & & & & \\
\hline Platelet count decreased & 3 & 25.0 & 1 & 8.3 & & & & \\
\hline Constipation & 2 & 16.7 & 1 & 8.3 & & & & \\
\hline Edema limbs & 2 & 16.7 & & & 1 & 8.3 & & \\
\hline Fatigue & & & 2 & 16.7 & 1 & 8.3 & & \\
\hline Alkaline phosphatase increased & & & & & 2 & 16.7 & & \\
\hline Anorexia & 1 & 8.3 & 1 & 8.3 & & & & \\
\hline Diarrhea & 2 & 16.7 & & & & & & \\
\hline Hypertension & & & 1 & 8.3 & 1 & 8.3 & & \\
\hline Alanine aminotransferase increase & & & & & 1 & 8.3 & & \\
\hline Anemia & & & 1 & 8.3 & & & & \\
\hline Blood bilirubin increased & & & 1 & 8.3 & & & & \\
\hline CD4 lymphocytes decreased & & & 1 & 8.3 & & & & \\
\hline Cough & & & 1 & 8.3 & & & & \\
\hline Dry skin & & & 1 & 8.3 & & & & \\
\hline Headache & & & 1 & 8.3 & & & & \\
\hline Hoarseness & & & 1 & 8.3 & & & & \\
\hline Hyperglycemia & & & & & 1 & 8.3 & & \\
\hline Lymphocyte count decreased & & & 1 & 8.3 & & & & \\
\hline Neutrophil count decreased & & & 1 & 8.3 & & & & \\
\hline Vomiting & 1 & 8.3 & & & & & & \\
\hline
\end{tabular}

Figures 
a. Median PFS - 2.4 months (95\% CI: 1.9 - 9.2 months)

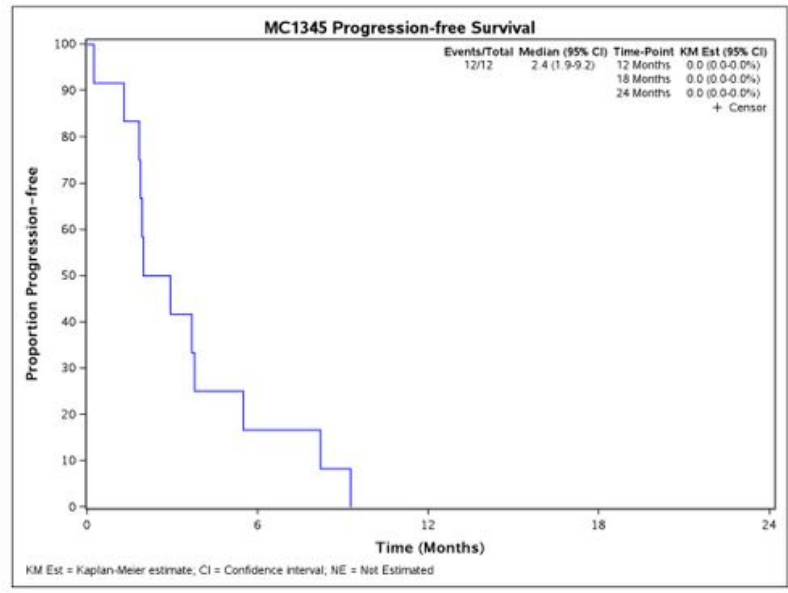

b. Median OS- 15.7 months (95\% CI: 6.1 - Not estimated)

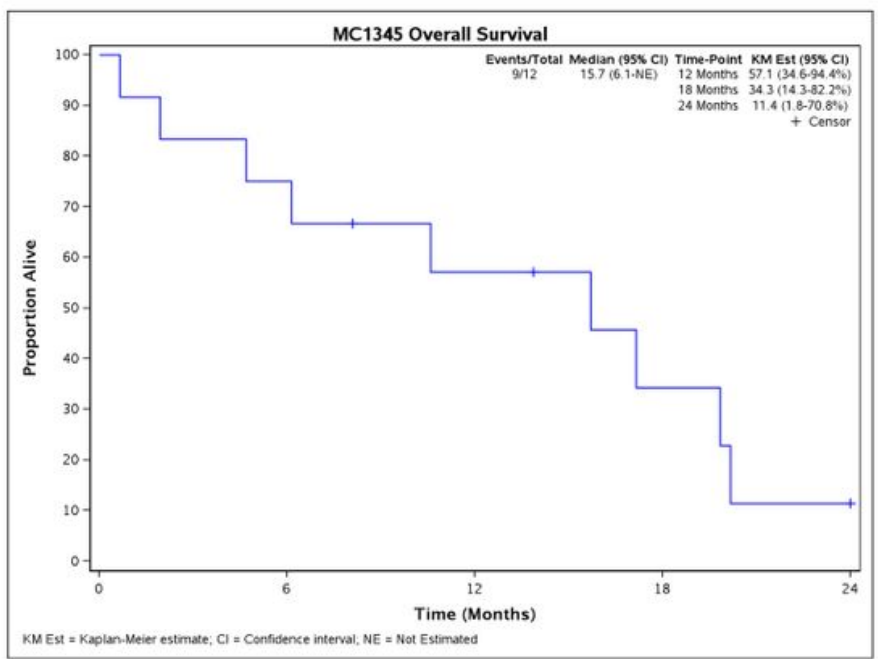

\section{Figure 1}

Kaplan-Meier estimates of progression free survival (PFS) and overall survival (OS). (a) Shows the PFS in patients with BTC who received ponatinib. (b) Shows OS for the same patient cohort.

\section{A. Spider plot presenting duration and change from baseline for tumors for each subject.}

Figure 2: Perœent change in tumor lesion size

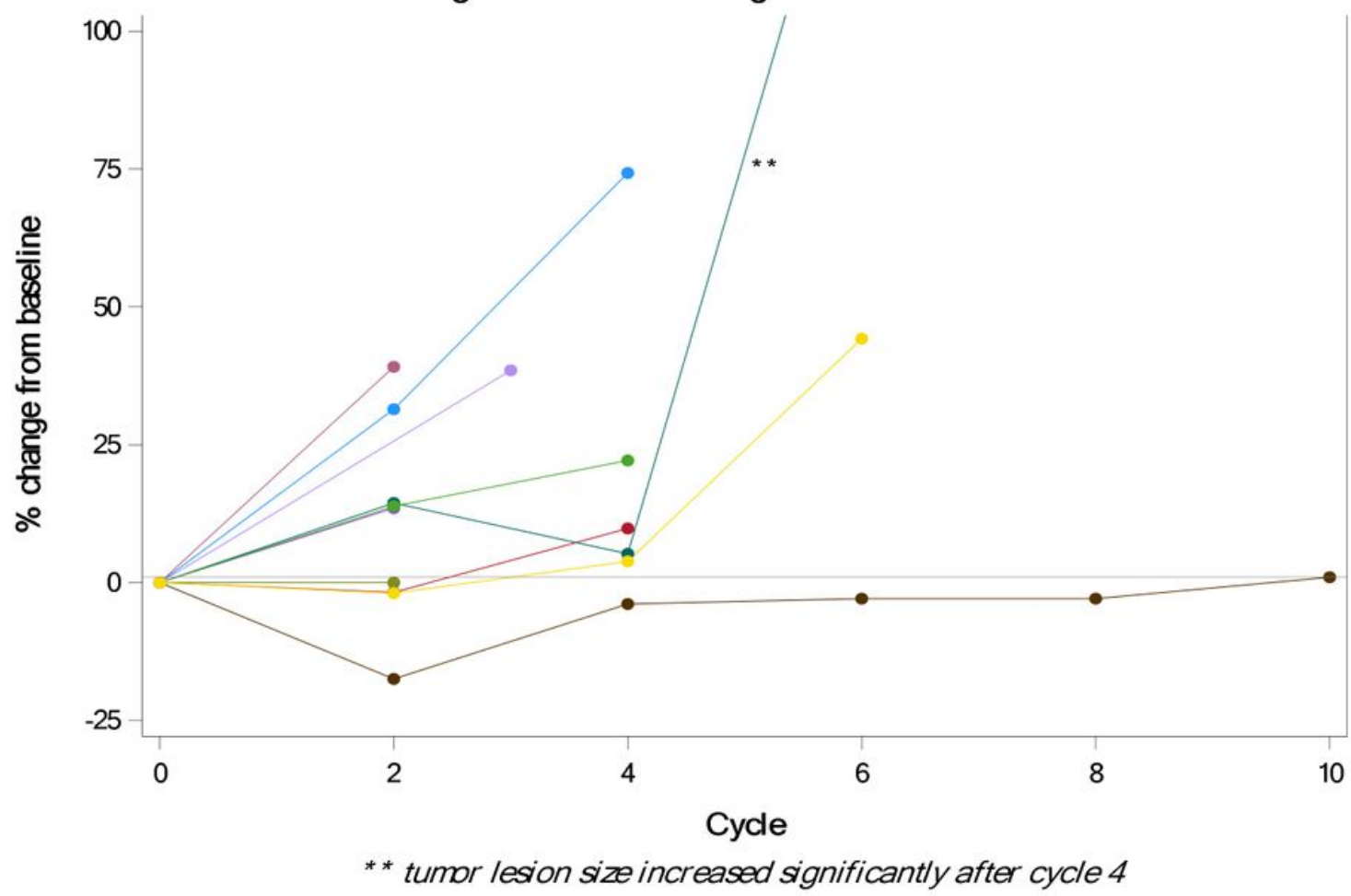

Figure 2 
Clinical response to ponatinib

\section{Supplementary Files}

This is a list of supplementary files associated with this preprint. Click to download.

- Supplementaryfiles.docx 